\title{
MANIFESTAÇÃO DE HEMANGIOENDOTELIOMA HEPÁTICO EM ADULTO
}

\author{
HEPATIC HEMANGIOENDOTHELIOMA IN ADULT
}

\author{
Aderivaldo Coelho de Andrade-TCBC-PI ${ }^{1}$; Ivo Canamary da Silveira Ribeiro ${ }^{2}$; \\ Álvaro José de Morais Araújo ${ }^{2}$
}

\section{INTRODUÇÃO}

O hemangioendotelioma hepático é um tumor vascular sintomático de estágio intermediário de malignidade, já que histopalologicamente é caracterizado como benigno, mas que pode apresentar-se de maneira agressiva, evoluindo com insuficiência cardíaca de alto débito, coagulopatia de consumo e anemia que aumentam significativamente a mortalidade desses pacientes ${ }^{1}$. Embora raro, é o tumor hepático mais frequiente no primeiro ano de vida, raramente observado acima dos três anos de idade ${ }^{1,2}$. Achados em adultos são extremamente raros, sendo descritos apenas dois casos até então, em um paciente de 18 anos e um outro de 56 $\operatorname{anos}^{2,3}$.

O objetivo deste trabalho é descrever um tumor hepático em paciente de 35 anos, com histopatológico caracterizando hemangioendotelioma infantil.

\section{RELATO DO CASO}

Mulher de 35 anos, dona de casa, natural do Maranhão, referia que há cerca de um ano evoluia com astenia, náuseas, plenitude pós-prandial, emagrecimento e aumento progressivo em hipocôndrio direito que a dificultava de desempenhar suas atividades corriqueiras. Ao exame físico, apresentava-se com bom estado geral, normocorada, anictérica, normotensa e com massa palpável, irregular a 12 centímetros do rebordo costal direito. A Tomografia Computadorizada revelou tumoração de $14,5 \times 11,5 \mathrm{~cm}$ no lobo direito hepático com zona de necrose (Figura 1). Os exames pré-operatórios apresentavam hematócrito $40 \%$, hemoglobina de $13,8 \mathrm{~g} / \mathrm{dl}$, plaquetas de $200000 / \mathrm{mm}^{3}$, leucócitos de $7200 / \mathrm{m}^{3}$, atividade de protrombina de $45 \%$ que melhorou após terapia com vitamina $\mathrm{K}$, com novo TAP de $95,4 \%$, bilirrubinas e transaminases normais. A paciente foi operada no dia 24 de junho de 2004.

No ato cirúrgico foi identificado volumoso tumor em toda extensão do lobo direito, com áreas irregulares e zona de necrose central, com risco iminente de ruptura. Foi realizada hepatectomia direita. O estudo histopatológico da lesão evidenciou um tumor composto por pequenos vasos com endotélio espesso e nos locais com lúmen vascular pequeno aparecem lesões sólidas, mitoses raras e sem significativo pleomorfismo celular que caracteriza um hemangioendotelioma hepático infantil.

A paciente teve boa evolução pós-operatória com alta no $9^{\circ}$ dia pós-operatório e atualmente após seis meses de cirurgia se encontra assintomática.

\section{DISCUSSÃO}

O hemangioendotelioma é uma entidade benigna, mas pode tornar-se agressiva, tendo sua evolução complicada por insuficiência cardíaca de alto debito, coagulopatia intravascular disseminada e sinais de hipertensão portal que muitas vezes se tornam intratáveis. No entanto, sua forma de apresentação mais comum é como massa abdominal palpável ou hepatomegalia ${ }^{1}$.

A maioria dos hemangioendoteliomas são diagnosticados na infância principalmente nos primeiros seis meses sendo que geralmente regridem espontaneamente sem a necessidade de intervenção ${ }^{2}$. Sua manifestação em adulto é extremamente rara, sendo descrita até então apenas dois casos, um desses com 18 anos e outro com 56 anos $^{2,3}$.

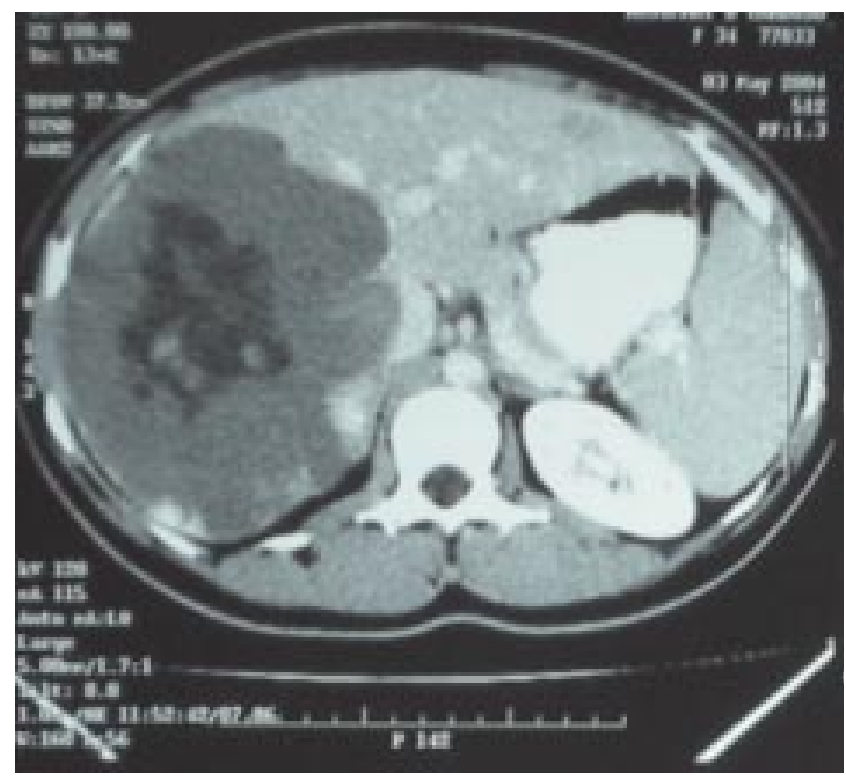

Figura 1 - Tomografia demonstrando grande tumor em lobo hepático direito com área de necrose central.

1. Cirurgião da Clinica Cirúrgica do Hospital Getúlio Vargas

2. Médicos formados pela Universidade Federal do Piauí.

Recebido em 13/06/2005

Aceito para publicação em 12/08/2005

Conflito de interesses: nenhum

Fonte de financiamento: nenhuma

Trabalho realizado na Clínica Cirúrgica do Hospital Getúlio Vargas - Universidade Federal do Piauí 
O diagnóstico clínico se dá pela presença de massa abdominal em hipocôndrio direito em crianças com menos de um ano, sendo a presença de hepatomegalia associada a insuficiência cardíaca, hemangiomas cutâneos, coagulopatia de consumo com trombocitopenia (síndrome de Kassabach-Merit) bastante sugestiva de hemangioendotelioma hepático infantil $^{4}$. A biopsia hepática é contra-indicada devido o alto risco de sangramento.

Em crianças, os hemangioendoteliomas devem ser tratados, inicialmente, de maneira conservadora, sendo reservado o tratamento cirúrgico para os casos de insuficiência cardíaca intratável e coagulopatia de consumo refratária ${ }^{4}$. Atualmente, tem-se utilizado o tratamento intervencionista por cateter através da oclusão arterial ou venosa associado com corticoterapia apresentando resultados bastantes significativos ${ }^{4}$.
Em nossa paciente, apesar do grande tamanho do tumor não havia nenhuma co-morbidade peculiar do hemangioendotelioma infantil como insuficiência cardíaca ou coagulopatia de consumo. Devido às grandes dimensões da lesão, associada a área de necrose com risco iminente de ruptura preferiu-se a intervenção cirúrgica cujo resultado foi satisfatório, evoluindo sem complicações no pós-operatório.

A historia natural dos hemangioendoteliomas infantis hepáticos habitualmente mostra crescimento rápido até a estabilização das lesões, que então sofrem regressão gradual. Após um período de tempo variável normalmente dentro de alguns meses, essas lesões deixam de existir ${ }^{5}$. Como existem poucos casos desses tumores em adultos, acredita-se que representem uma doença residual de crescimento lento.

\begin{abstract}
Hepatic hemangioendothelioma is the most frequent hepatic tumor in infancy, but rarely detected in adults. This tumor can cause vascular lesions that can act as arteriovenous fistulas and produce life-threatening high output congestive heart failure with respiratory compromise. We report a case of a 35 years-old woman who developed nausea, vomiting, weight lost and abdominal mass in which the pathological examination of the hepatic lesion showed a infantile hepatic hamangioendothelioma. This is the third case in adult patients described in the literature (Rev. Col. Bras. Cir. 2007; 34(2): 133-134).
\end{abstract}

Key words: Hemangioendothelioma; Liver neoplasms.

\title{
REFERÊNCIAS
}

1. Dachaman AH, Lichtenstein JE, Friendman AC, Hartman DS. Infantile hemangioendothelioma of the liver: a radiologicpathologic-clinical correlation. AJR Am J Roentegenol. 1983;140(6):1091-4.

2. Diment J, Yurim O, Pappo O. Infantile Hemangioendothelioma of the liver in an adult. Arch Pathol Lab Med. 2001;125(7):931-2.

3. Selby DM, Stocker JT, Waclawiw MA, Hitchcock CL, Ishak KG. Infantile hemangioendothelioma of liver. Hepatology. 1994;20(1 Pt 1):39-45.

4. Daller AJ, Bueno J, Gutierrez J, Dvorchik I, Towbin RB, Dickman PS, Mazariegos G, Reyes J. Hepatic hemangioendothelioma: clinical experience and management strategy. J Pediatr Surg. 1999;34(1):98-105; discussion 105-6.

5. Kaniklides C, Dimopoulos PA, Bajic D. Infantile hemangioendothelioma. A case report. Acta Radiol. 2000;41(2):161-4
Como citar este artigo:

Andrade AC, Ribeiro ICS, Araújo AJM. Hemangioendotelioma hepático em adulto: relato de caso. Rev Col Bras Cir. [periódico na Internet] 2007; 34(2). Disponível em URL: http://www.scielo.br/ $\mathrm{rcbc}$

Endereço para correspondência:

Aderivaldo Coelho de Andrade

R.Pedro Vasconcelos, $1790 / 501$

Bairro - Noivos

64045-050 - Teresina - PI

Email: domderi@hotmail.com

Tel: 086-9982-3056 e 086-234-9434 